\title{
Interferometry with ALOS-2 full-aperture ScanSAR data
}

Cunren Liang

Jet Propulsion Laboratory, California Institute of Technology, Pasadena, CA, USA

cunrenl@caltech.edu

Eric J. Fielding

Jet Propulsion Laboratory, California Institute of Technology, Pasadena, CA, USA

eric.j.fielding@jpl.nasa.gov

This is a post-print submitted to EarthArXiv before final IEEE editing and formatting. The paper is published as:

C. Liang and E. J. Fielding, "Interferometry with ALOS-2 full-aperture ScanSAR data," IEEE Transactions on

Geoscience and Remote Sensing, vol. 55, no. 5, pp. 2739-2750, May 2017. DOI: 10.1109/TGRS.2017.2653190 


\title{
Interferometry with ALOS-2 full-aperture ScanSAR data
}

\author{
Cunren Liang and Eric J. Fielding ${ }^{1}$
}

\begin{abstract}
ALOS-2 is designed to routinely acquire both ScanSAR and stripmap data. In this paper, we present a special multi-band bandpass filter (MBF) to remove azimuth nonoverlap spectra for the interferometric processing of ALOS-2 full-aperture ScanSAR product. As required by the MBF, we estimate the important ScanSAR system parameters and the start times of raw bursts using ALOS-2 full-aperture ScanSAR image. The resulting MBF can remove the non-overlap spectra caused by both Doppler centroid frequency difference and burst misalignment. It can be used in ScanSAR-ScanSAR interferometry, as well as ScanSAR-stripmap interferometry. Based on the MBF, we propose a single processing workflow that is able to implement both ScanSAR-ScanSAR interferometry and ScanSAR-stripmap interferometry. Finally, we present example interferograms of the 2015 Gorkha earthquake in Nepal processed using the proposed processing workflow. The interferograms are significantly improved after applying MBF.
\end{abstract}

Index Terms - scanning synthetic aperture radar (ScanSAR), full-aperture, interferometry, ALOS-2, earthquake.

\section{Introduction}

Scanning synthetic aperture radar (ScanSAR) [1], [2] has been widely used by recent spaceborne synthetic aperture radar (SAR) systems to acquire wide-swath images. In ScanSAR mode, the SAR antenna cyclically points to several subswaths. In each cycle, the SAR system collects a group of echoes for each subswath. The group of echoes is called a burst. By repeating this cycle over several subswaths, a wide-swath image can be acquired. Interferometry with ScanSAR data requires that bursts of master and slave acquisitions should overlap. Besides stripmap mode, ScanSAR mode is also routinely used in the global acquisition strategy [5] of the Japan Aerospace Exploration Agency (JAXA) Advanced Land Observation Satellite 2 (ALOS-2) mission [3], [4] launched on May 24, 2014. Since February 2015, the burst overlap of ALOS-2 ScanSAR mode has been under control. Interferometry with ALOS-2 ScanSAR data is interesting for geophysical research considering the wide-swath coverage, as well as the highly coherent signals thanks to the good burst overlap, short baseline and long wavelength of L-band. Furthermore, ScanSAR-stripmap interferometry can also benefit geophysical research by increasing the temporal sampling of InSAR data stacks in time series analysis.

JAXA does not release ALOS-2 raw data. For ScanSAR data, two kinds of focused product are distributed. One kind is focused burst by burst, and the other one is focused using the full-aperture algorithm [6]. For interferometry [7], the two kinds of products correspond to two kinds of processing approaches which can be called burstby-burst approach and full-aperture approach [6], [8]-[11]. The first one is usually

\footnotetext{
${ }^{1}$ The authors are with the Jet Propulsion Laboratory, California Institute of Technology, Pasadena, CA 91109 USA.

Copyright 2016 California Institute of Technology. U.S. Government sponsorship acknowledged.
} 
considered to be a more standard approach. Its major advantage is the smaller data volume. Its critical step is accurate coregistration that uses the commonly accepted geometrical coregistration [12] followed by spectral diversity coregistration [13]. However, according to our experiments, while geometrical coregistration is very accurate for most current spaceborne SAR products, it is not for ALOS-2 products due to unknown reasons. It is also not accurate enough for the coregistration of stripmap-stripmap interferometry. The big coregistration error after geometrical coregistration may cause problems for applying spectral diversity coregistration. Processing time is not necessarily an advantage of this approach, as the overall coregistration stage including geometrical coregistration, spectral diversity coregistration and the repeated InSAR steps requires a lot of time.

The major advantage of full-aperture processing is that existing stripmap InSAR processors, e.g. ROI_pac [14], GMTSAR [11] and ISCE [15], can still be used. It has been shown that in general, the cross-correlation coregistration method usually available in a stripmap InSAR processor can also be used to coregister full-aperture images [16]. Preliminary ALOS-2 ScanSAR interferograms processed using this approach [17], [18] have greatly contributed to the research of the April 25, 2015 Gorkha earthquake in Nepal [19], [20], which has attracted wide interest among the geophysical community. These early interferograms were processed by simply putting full-aperture products into a stripmap InSAR processor. Potential problems, such as non-overlap spectra, were not considered. It is important to step forward and develop more sophisticated methods to process the full-aperture product. However, there are difficulties in doing this. First of all, some important parameters of ALOS-2 ScanSAR mode are not officially released by JAXA. Second, the full-aperture ScanSAR image is a continuous stripmap-like image, and the removal of non-overlap spectra is complicated.

In the following sections, we present our methods for interferometry with ALOS-2 full-aperture ScanSAR product with a focus on the removal of azimuth non-overlap spectra using a special multi-band bandpass filter (MBF), along with the estimation of the parameters required to implement the MBF. A processing workflow based on the MBF is presented for both ScanSAR-ScanSAR interferometry and ScanSARstripmap interferometry. This processing workflow is used to process interferograms of the 2015 Gorkha earthquake in Nepal.

II. Overview of the Proposed Processing Workflow for Both ScanSAR-ScanSAR Interferometry and ScanSAR-stripmap Interferometry

For interferometric processing of ScanSAR data, the full-aperture approach is nearly the same as stripmap InSAR processing. The major differences are filling burst gaps with zero echoes before focusing [6] and removing non-overlap spectrum caused by burst misalignment. In [21], ScanSAR and its interferometric processing are comprehensively discussed.

The full-aperture approach can be used to process ALOS-2 full-aperture ScanSAR product. With the full-aperture product, while filling burst gaps with zero echoes and focusing have already been done, the removal of non-overlap spectra caused by Doppler centroid frequency difference and burst misalignment remains an issue. Furthermore, some important parameters including burst length, burst cycle length, and the start times of raw bursts are needed in the removal of non-overlap spectra, but 
are not provided in the full-aperture product. Our objective is to first estimate these important parameters from the product, and then design an MBF to remove the nonoverlap spectra. The resulting MBF should also be applicable to stripmap data in ScanSAR-stripmap interferometry. For stripmap data, the non-overlap spectra corresponding to ScanSAR burst gaps and caused by Doppler centroid frequency difference should be removed.

The overall processing workflow for forming an interferogram is shown in Fig. 1. The remaining steps are the same as those of stripmap InSAR processing. We use the JPL ISCE [15] for the remaining steps. Before implementing the processing workflow, we estimate the burst length and burst cycle length using the full-aperture image. The estimation results are saved in our processor. In the processing workflow, we estimate the start times of raw bursts using the full-aperture image. In this step, we also deal with the problem of burst length change of subswath 5 as discussed in Section III-D. ALOS-2 uses three wavelengths and several range bandwidths [4]. For ScanSARstripmap interferometry, the range band of stripmap data is usually different from that of ScanSAR data. Even for ScanSAR-ScanSAR interferometry, the range bands of the pair may be different. Therefore, range filtering is necessary to keep only the common range spectrum. Furthermore, the range and azimuth sample sizes of the pair may be different. We resample one of the images to equalize the sample sizes to enable the subsequent cross-correlation coregistration. The burst overlap is then calculated. With the required parameters available at this point, we use the MBF to remove nonoverlap spectra caused by both Doppler centroid frequency difference and burst misalignment. We then use the cross-correlation method to coregister the pair [16]. Now a subswath interferogram of a frame can be formed. After forming interferograms of all the subswaths of all the frames, we mosaic them.

Before the mosaicking, we need to know the precise offsets between subswaths and frames. A comparison of the offsets estimated by coregistration and calculated from the related parameters shows that the difference of the two kinds of offsets can be larger than one sample. Instead of using offsets calculated from related parameters, we use offsets estimated by coregistration for subswath and frame mosaicking.

Subswath or frame mosaicking basically involves resampling and putting the resampled subswath or frame in its correct location according to the precise offsets. We first mosaic subswath interferograms to form the interferogram of a whole frame. For adjacent subswath interferograms, we don't find an obvious phase difference, and therefore we don't apply phase adjustment between subswath interferograms. Note that even for ScanSAR-stripmap interferometry, subswath mosaicking is necessary, because for ALOS-2, the stripmap acquisition overlaps with two subswaths of the ScanSAR acquisition in most of the cases. We then mosaic the frame interferograms to form the interferogram of a long track. For adjacent frame interferograms, we find an obvious phase difference. This is probably caused by different focusing conditions of adjacent frames. We fit a low order polynomial to the phase difference and subtract it from the interferogram of next frame.

In our prototype implementation, the whole processing workflow can be completed by calling the same command for both ScanSAR-ScanSAR interferometry and ScanSAR-stripmap interferometry, even for multiple frames.

In the following sections, we discuss the estimation of the important parameters and the MBF. 


\section{The Estimation of Burst Length and Burst Cycle Length}

\section{A. The Separation of the Burst Signals in Full-Aperture Product}

The overall geometrical and spectral properties of full-aperture ScanSAR image are similar to those of stripmap image. Signals from different bursts are mixed together in both time and frequency domains. Usually, a burst can also be called a look for a target [21]. Therefore, ScanSAR data focused using full-aperture algorithm is not single look complex (SLC), but essentially multiple look complex (MLC), which is the reason why we have been calling the focused image full-aperture image, rather than full-aperture SLC. To estimate burst length and burst cycle length from this continuous stripmap-like image, first of all, different bursts should be separated in either time or frequency domain. Fortunately, the way the burst signals are mixed is in essence simple addition, which should make the separation easier.

For a target, the signal of one burst after focusing can be represented by [8]

$$
s_{f}(\eta)=T_{B} \operatorname{sinc}\left(K_{a} T_{B} \eta\right) \cdot \exp \left\{j \pi K_{a} \eta\left(\eta-2 \eta_{c}\right)\right\}
$$

where $T_{B}$ is the burst length, $\eta$ is the azimuth time, $\eta_{c}$ is the burst center time for the target, and $K_{a}\left(K_{a}>0\right)$ is the azimuth FM rate. We have ignored the range of closest approach, the antenna pattern, the weighting applied in the focusing and the complex constant modeling the backscattering characteristic. A full-aperture image is a superposition of $N_{L}$ such single burst signals, where $N_{L}$ is the number of looks of the ScanSAR mode. To separate the bursts, we multiply the full-aperture image in azimuth direction by the following quadratic phase

$$
p(\eta)=\exp \left\{-j \pi K_{a}(\eta+\Delta \eta)^{2}\right\}
$$

where we have used $\Delta \eta$ to denote the location of the phase. This is known as deramp in SPECtral ANalysis (SPECAN) algorithm [8]. After deramping, the signal becomes

$$
s_{d}(\eta)=T_{B} \operatorname{sinc}\left(K_{a} T_{B} \eta\right) \cdot \exp \left\{j 2 \pi K_{a} \eta\left(-\eta_{c}-\Delta \eta\right)+j C\right\}
$$

where $C$ is the remaining constant phase. It is important to note that before deramping, the bandwidth of $s_{f}(\eta)$ may be much larger than $K_{a} T_{B}$, and the spectrum may not be a rectangle due to the quadratic phase term in the signal [22]. After deramping, the bandwidth of $s_{d}(\eta)$ is $K_{a} T_{B}$, and the spectrum becomes a rectangle. The frequency center is shifted from $-K_{a} \eta_{c}$ to $-K_{a}\left(\eta_{c}+\Delta \eta\right)$. This implies that, for the same target, the frequency center is shifted by $-K_{a} \Delta \eta$, regardless of the burst the signal is from. That is, the frequency separation $K_{a} T_{C}$ between different bursts for a target remains the same, where $T_{C}$ is the burst cycle length. Note that we have been using relative time (variable $\eta$ ) in this single target analysis, where the zero time $(\eta=0)$ corresponds to the zero Doppler frequency of the target under discussion. For multiple targets imaged by the same burst, the amount of frequency shift is different for different targets, and the spectra of all targets are shifted to the same band, which has been discussed in other publications.

An example is shown in Fig. 2. Note that Fig. 2(b) is not a single target spectrum as usually drawn in other publications, but spectra of many targets. The number of bursts in this figure is also not necessarily the number of looks $N_{L}$ of the ScanSAR mode. The main reason why the spectrum is weighted is that fewer targets of edge bursts are involved in the fast Fourier transform (FFT). Now it is very clear that the burst length and burst cycle length can be calculated according to the time-frequency relationship 
of the azimuth SAR signal. However, the precise estimation of burst length and burst cycle length is not that easy. While we find that the estimation is very precise for simulated signals, it is not for real data. The rectangle shown in Fig. 2(b) is actually a trapezoid for this real data case.

\section{B. Practical Estimation Method}

Since the shape of the spectrum shown in Fig. 2(b) is more like a trapezoid rather than a rectangle, the burst bandwidth slowly varies from the bottom to the top of the trapezoid. It is important to determine at what height the bandwidth should be used in order to get a more reliable estimation. To determine this height, we first do the estimation using ScanSAR data extracted from ALOS-1 stripmap raw data. We can easily get ScanSAR data from ALOS-1 stripmap raw data by removing raw echoes corresponding to burst gaps.

Specifically, we use the following steps to determine the optimum height.

(1) Focus the ScanSAR data extracted from ALOS-1 stripmap raw data using full-aperture algorithm.

(2) Read in a focused data block. To use all the signals collected by a burst, the number of lines we use is equivalent to the length of full aperture. We use a number of columns to get a more regular spectrum.

(3) In azimuth, deramp and do FFT on the data block. Average the spectra over the columns to get a regular azimuth spectrum, like the one shown in Fig. 2(b).

(4) Pick up the burst that is fully covered by the data block. Calculate the burst length according to the following relationship between time and frequency

$$
T_{B}=\frac{W_{B}}{K_{a}}
$$

where $W_{B}$ is the burst bandwidth read from the estimated azimuth spectrum. As discussed earlier, the burst bandwidth slowly varies from the bottom to the top of the trapezoid. For the ScanSAR data extracted from ALOS-1 raw stripmap data, we know beforehand the real $T_{B}$, and thus can determine at what height the bandwidth should be used according to (4).

We use the same steps (except the first one) to estimate the burst length and burst cycle length of ALOS-2 ScanSAR data. For these steps, the only difference is that now we know at what height the bandwidth should be used. The estimation of burst cycle length is not affect by the trapezoid shape of the spectrum, as we can always use the bandwidth spanning the center frequencies of two adjacent bursts.

To get more precise estimation results, we follow these steps to do a number of estimates over the whole image. We then average these estimates to get the burst length and burst cycle length. For burst cycle length, we can reduce the estimation error by comparing the actual time span between the first and last bursts calculated using their spectra and the time span calculated using estimated burst cycle length. For the same ScanSAR acquisition, the burst cycle lengths of all the subswaths should be the same, and therefore the burst cycle length can be further improved by averaging all the subswath burst cycle lengths.

\section{Estimation Results of an ALOS-2 ScanSAR Mode}


ALOS-2 can acquire data in several different ScanSAR modes [4], [23]. Here we present the estimated parameters of WBD mode (ScanSAR nominal [14MHz] mode Dual polarization, Beam No: W2), which is mostly used.

The estimation results are listed in Table I. These values are expressed as number of each subswath's 1/PRF. We then represent them as number of subswath 1's 1/PRF, which is shown in Table II. As we can see from this table, the burst length increases with subswath number. We guess that this is to make all subswath azimuth bandwidths approximately the same, because azimuth FM rate decreases as slant range increases. To verify this, we calculate the swath center bandwidth of each subswath using each subswath's burst length and subswath 3's burst length for frame 0550 acquired on Aug. 9, 2014. The results are shown in Fig. 3, from which we can see the effect of increasing burst length.

The error of the estimation results may cause resolution loss or incomplete nonoverlap spectrum removal after MBF is applied. We take subswath 3 of frame 0550 acquired on Aug. 9, 2014 as an example. The estimated burst length is 358 1/PRF, which corresponds to a bandwidth of $81.26 \mathrm{~Hz}$ in the subswath center. Even with an estimation error of $10 \mathrm{1} / \mathrm{PRF}$, it only corresponds to a bandwidth error of $2.27 \mathrm{~Hz}$, which should not cause severe problem for MBF. According to the experience with ALOS-1 data, the accuracy of the burst length estimation can be on the order of several 1/PRF. The accuracy of burst cycle length estimation can be as high as on the order of several hundredths of $1 / \mathrm{PRF}$.

\section{The Solution for the Burst Length Change of Subswath 5}

According to one of the reviewers of an early version of this manuscript and our data processing experience, the burst length of subswath 5 is usually subject to small changes mostly depending on the acquisition area. This change affects burst cycle length. In the determination of start times of raw bursts, which will be discussed in the next section, for each subswath we estimate the start time of a raw burst and infer the start times of other raw bursts using burst cycle length. Although the change of burst cycle length is small, the accumulating effect in the determination of start times of raw bursts cannot be ignored.

This problem can be simply solved as follows. We estimate the start time of a raw burst at the start of the image and then calculate the start time of another raw burst at the end of the image using current burst cycle length. We then estimate the start time of the raw burst at the end of the image. The difference of the two start times can be used to update the burst cycle length.

\section{Automatic Estimation of the Start Times of Raw Bursts}

There is no need to do the estimation of burst length and burst cycle length each time we process data of same ScanSAR mode. The estimation can be done beforehand only once, and therefore it can be done manually. This is not the case for the start times of raw bursts, as they are different for different acquisitions. The estimation of the start times of raw bursts should be done automatically.

Like the estimation of burst length and burst cycle length, we also use the azimuth spectrum to estimate the start times of raw bursts. However, special attention should be paid to the spectrum to be used. From Fig. 2(b), we can see that burst signals outside of the PRF interval are shifted to the PRF interval due to aliasing. This is not a 
severe problem for the manual estimation of burst length and burst cycle length, as we can easily identify it. For the automatic estimation of the start times of raw bursts, however, it is not straightforward to identify it by programming. We should try to avoid it. One method is to properly determine the number of lines to be used in FFT.

\section{A. The Number of Lines to Be Used in FFT}

For one line or one target in the full-aperture image, we never have to consider the aliasing effect, as the spectrum is within a PRF interval before and after deramping. In this case (hereafter referred to as one-line case), the number of bursts involved roughly represents the number of looks $N_{L}$ of the ScanSAR mode. But we cannot use only one line to calculate the spectrum. Starting from the first line to be used in the FFT, more bursts may be involved in the FFT as we use more lines, compared with the one-line case. This potentially results in the aliasing effect.

In determining the number of lines that should be used in the FFT, we find that the number of lines $N_{l}$ corresponding to the burst cycle length $T_{C}$ is a critical number. If we use $N_{l}$, at most one additional burst will be involved in the FFT, compared with the one-line case. This greatly reduces the risk of aliasing effect. If we use a number that is larger than $N_{l}$, even more bursts may be involved in the FFT. On the other hand, if we use a number that is smaller than $N_{l}$, the number of bursts involved is not necessarily reduced compared with the case of $N_{l}$. Besides, we find that $N_{l}$ is a reasonable length for FFT for ALOS-2 ScanSAR mode. For the FFT length, we can use the smallest power of two that's greater or equal to $N_{l}$.

\section{B. Practical Estimation Method}

For practical estimation of the start times of raw bursts, we use the following steps.

(1) Read in a data block of full-aperture image. The number of lines of the data block is $N_{l}$. We also use a number of columns to get a more regular spectrum.

(2) In azimuth, deramp and do FFT on the data block. Average the spectra over the columns to get a regular azimuth spectrum, like the one shown in Fig. 4(a).

(3) Calculate the correlation between the magnitude of the spectrum and the magnitude of an artificial burst spectrum with bandwidth equal to $K_{a} T_{B}$. There are a number of peaks representing the frequency centers of the bursts, as shown in Fig. 4(b). It is easy to find the location of the peak within one burst cycle.

(4) Using burst length and the location of the peak within one burst cycle, calculate the start time of the corresponding raw burst according to the timefrequency relationship (4). With the start time of one raw burst determined, start times of other bursts can be inferred using burst cycle length $T_{C}$.

Since we use the center of the burst spectrum in the calculation, the result is not affected by the trapezoid shape of the spectrum. Like the error in the estimation results of burst length and burst cycle length, the error of the estimation result may cause resolution loss or incomplete non-overlap spectrum removal after MBF is applied. However, as mentioned earlier, even with an estimation error of 10 1/PRF, it should not cause severe problem for MBF. Experience with the processing of a large amount of data shows that this method is robust and can be fully automated.

Now that we have the start times of raw bursts, the burst overlap of an InSAR pair can be determined using the azimuth offset of the InSAR pair which can be calculated using the geometrical coregistration that is used in burst-by-burst approach. 
Both Doppler centroid frequency difference and burst misalignment cause nonoverlap spectra, the removal of which has been discussed in the early publications [7], [21]. One method is to remove the non-overlap raw echoes from the bursts before focusing to account for burst misalignment. Another method is to remove the nonoverlap spectra by filtering, which is mainly focused on single burst filtering in the burst-by-burst ScanSAR interferometric processing. For the non-overlap spectra removal of ALOS-2 full-aperture product, the first method is obviously not available since it requires raw data. While the second method can be used, single burst filtering method is inefficient for this multiple burst problem. For the ScanSAR mode discussed in section III-C, the number of looks is nearly 5 , so each target needs to be filtered 5 times if single burst filtering method is used. Note that typical subswath image file size ranges from 4 to 6 GB for an ALOS-2 ScanSAR frame. Obviously this is an inefficient method. Our solution is to design an MBF that only needs to filter each target once, and at the same time removes all the non-overlap spectra. Such an MBF is not straightforward, and several issues have to be considered.

\section{A. The Effect of Doppler Centroid Frequency Difference and Burst Misalignment}

Before we present our MBF, we should make it clear how Doppler centroid frequency difference and burst misalignment affect the azimuth spectrum of the InSAR pair. As shown in Fig. 5, Doppler centroid frequency difference will reduce the full azimuth bandwidth of the InSAR pair. With Doppler centroid frequency difference, the number of looks of the InSAR pair becomes

$$
N_{L}=\frac{\left(T_{A}-T_{D}\right)-T_{B}}{T_{C}}
$$

where $T_{A}$ is the full aperture length, and $T_{D}$ is the time difference corresponding to the Doppler centroid frequency difference. Burst misalignment does not affect the full azimuth bandwidth, but it will reduce the burst bandwidth of the InSAR pair. On the other hand, burst cycle length remains the same. Considering also the burst misalignment, the number of looks of the InSAR pair becomes

$$
N_{L}=\frac{\left(T_{A}-T_{D}\right)-\left(T_{B}-T_{M}\right)}{T_{C}}
$$

where $T_{M}$ is the burst misalignment.

For interferometry, the master and slave spectra of a target are correlated in the frequency domain as shown in Fig. 6 [24]. If there is no Doppler centroid frequency difference between the InSAR pair, the set of master bursts illuminating the target exactly corresponds to the set of slave bursts illuminating the target. The gray baseband spectrum shown in Fig. 6(a) is a superposition of spectra of $N_{L}$ single burst interferograms, each of which is formed by a pair of corresponding (coherent) master and slave bursts. Only the gray baseband spectrum is the signal we need in interferometry. If there is Doppler centroid frequency difference between the InSAR pair, the two sets of bursts may be different. An example is shown in Fig. 5(a). For the azimuth spectra of a target indicated by vertical line $A A^{\prime}$ in Fig. 5(a), only two of the three blue bursts correspond to two of the three green bursts (Here we only consider complete bursts. Note also that in Fig. 5(a), there is also burst misalignment.). In this case, as shown in Fig. 6(b), only the coherent burst pairs inside the rectangle 
contribute to the gray baseband spectrum. This is another way of explaining the reduction of the number of looks due to Doppler centroid frequency difference. In any case, only coherent burst pairs contribute to the gray baseband spectrum which will be extracted in the subsequent azimuth sample averaging. As a conclusion of the analysis, if there is Doppler centroid frequency difference, doing interferometry without removing the non-overlap spectrum caused by Doppler centroid frequency difference beforehand will not affect the quality of the final interferogram.

However, non-overlap spectrum caused by burst misalignment will affect the quality of the final interferogram. For the example shown in Fig. 6(c), although the gray spectrum looks like the one in Fig. 6(a), only the part on the left side of vertical axis is not contaminated by non-overlap spectrum. This is similar to the case of a stripmap InSAR pair with different Doppler centroid frequencies.

\section{B. $M B F$}

While Doppler centroid frequency difference will not affect the quality of the final interferogram, we find that considering the removal of non-overlap spectrum caused by it will not add too much complexity and computation to our MBF. For our implementation, we choose to add this function to our MBF. This function is also used in the experiments in section 9. Therefore, the resulting MBF can remove the non-overlap spectra caused by both Doppler centroid frequency difference and burst misalignment.

To design the MBF, we have to solve three problems. First, how many image samples should be used in the FFT? Second, how many passbands the filter should have? Third, where to start on the continuous stripmap-like full-aperture image? For the number of image samples to be used in the FFT, we can still use $N_{l}$. The actual number can be extended considering the filter length. For the number of passbands of the filter, we can determine it considering our previous discussions on the number of looks. The only problem left is where to start.

In Fig. 5(a), the overlap spectrum denoted by black can be seen as the spectrum of a new ScanSAR mode which is also the effective ScanSAR mode for the InSAR pair (hereafter referred to as effective ScanSAR mode). For the starting sample of the data block to be filtered by MBF, we can consider the starting or ending sample of a burst of the new ScanSAR mode (hereafter referred to as effective burst). If we choose the ending sample of an effective burst and the data block to be filtered is indicated by $T_{C}$ as shown in Fig. 5(a), for the acquisition with larger Doppler centroid frequency, bursts $B_{1}-B_{4}$ will be involved in the filtering, and only the overlap spectra of bursts $B_{3}$ and $B_{4}$ should be kept in the filtering. For the acquisition with smaller Doppler centroid frequency, passbands should be set for the two bursts corresponding to bursts $B_{3}$ and $B_{4}$. Here we call the two bursts $B_{3}^{\prime}$ and $B_{4}^{\prime}$. However, for burst $B_{4}^{\prime}$, within the time span of $T_{C}$ as shown in Fig. 5(a), its duration is longer than that of burst $B_{4}$. This is because the Doppler centroid frequency of this acquisition is smaller, and therefore the lower limit of the spectrum is smaller. As a result, if we set a passband for burst $B_{4}^{\prime}$ within $T_{C}$, part of non-overlap spectrum (on the left side of $T_{C}$ ) of $B_{4}^{\prime}$ remains after filtering. Therefore, two filters are required to completely remove the non-overlap spectrum of $B_{4}^{\prime}$. The first filter is for the first part (on the left side of $T_{C}$ ) of burst $B_{4}^{\prime}$ where burst $B_{4}$ cannot reach and there is no overlap between burst $B_{4}$ and $B_{4}^{\prime}$, and the other filter is for the remaining part (on the right side of $T_{C}$ ) of burst $B_{4}^{\prime}$. To avoid this problem, we should choose the starting sample of the effective burst for the acquisition with smaller Doppler centroid frequency. 
As a summary for the starting sample of the data block to be filtered by MBF, if Doppler centroid frequency is larger, the starting sample should be the ending sample of the effective burst; otherwise the starting sample should be the starting sample of the effective burst. In this way, within the time span of $T_{C}$ as shown in Fig. 5(a), we actually remove the non-overlap spectrum caused by Doppler centroid frequency difference by not setting passbands for the bursts whose spectra are outside of the common band that depends on Doppler centroid frequency difference.

To filter the full-aperture image, we locate the data block to be filtered. We deramp the data block using equation (2). We then filter the deramped data block using MBF. After filtering, the data block is reramped. This process is repeated until the whole image is filtered.

Note that this MBF can also be used to filter stripmap SLCs as shown in Fig. 5(b), which enables ScanSAR-stripmap interferometry.

\section{Experiments with ALOS-1 Raw Data}

We first examine what we have discussed about MBF in Section V by simulation experiments using ALOS-1 raw data acquired in stripmap mode, from which simulated ScanSAR data are extracted. The parameters of the ScanSAR InSAR pair are listed in Table III. We focus the extracted ScanSAR data using full-aperture algorithm and remove the non-overlap spectra using different methods.

The resulting interferograms are shown in Fig. 7. The azimuth size of the interferograms is equal to burst cycle length. The approximate number of looks of the effective ScanSAR mode is 1.69. This means there are two kinds of area within a burst cycle. The numbers of looks are one and two for the two kinds of area, respectively. In Fig. 7, the numbers of looks of the upper and lower areas are one and two, respectively. Fig. 7(a) is the original interferogram that is contaminated by the noise resulting from the non-overlap spectra caused by both Doppler centroid frequency difference and burst misalignment. We remove the non-overlap spectrum caused by burst misalignment using MBF and get the interferogram shown in Fig. 7(b), the noise of which is obviously reduced.

Then we try four different methods of removing non-overlap spectrum caused by Doppler centroid frequency difference. In the meantime, the non-overlap spectrum caused by burst misalignment is also removed. In Section V-B, we suggest that for the starting sample of the data block to be filtered by MBF, if Doppler centroid frequency is larger, the starting sample should be the ending sample of the effective burst; otherwise the starting sample should be the starting sample of the effective burst. The first method does the opposite. That is, if Doppler centroid frequency is larger, the starting sample is the starting sample of the effective burst; otherwise the starting sample is the ending sample of the effective burst. The second method always starts from the ending sample of the effective burst, while the third method always starts from the starting sample of the effective burst. The last method chooses starting sample in the suggested way.

The resulting four interferograms are shown in Fig. 7(c)-(f), respectively. In the upper 1-look area of the interferograms shown in Fig. 7(c)-(e), the non-overlap spectrum caused by Doppler centroid frequency difference is not completely removed. In Fig. 7(c), non-overlap spectra of both acquisitions in this area are not completely removed, while in Fig. 7(d) and Fig. 7(e), non-overlap spectrum of one of the acquisitions in 
this area is not completely removed. In Fig. 7(f), non-overlap spectra of both acquisitions in this area are completely removed. Therefore, we can see that, in this area, Fig. 7(c) is noisier than Fig. 7(d) and Fig. 7(e) which are noisier than Fig. 7(f). This is exactly because of the different starting samples chosen by different methods. On the other hand, the non-overlap spectrum in the lower 2-look area is completely removed for all cases. To further check the MBF filtering result shown in Fig. 7(f), we process data of the effective ScanSAR mode starting from raw data, and the result is shown in Fig. 7(g). By comparing Fig. 7(f) and Fig. 7(g), we can see that there is a small noisy strip between the 1-look and 2-look areas in Fig. 7(f). This is because of the signals with lengths smaller than burst length that are not considered in our MBF. Apart from this, no big difference is observed between the two interferograms.

The interferograms are then averaged and downsampled in the time domain. The results are shown in Fig. 8. The area shown in Fig. 7 is indicated by the black rectangle in Fig. 8(a). Despite the differences of the noise level of Fig. 7(b)-(f), Fig. 8(b)-(f) are nearly the same. This is because that the differences of the noise level of Fig. 7(b)-(f) are caused by different amounts of remaining non-overlap spectrum that is caused by Doppler centroid frequency difference. As analyzed in Section 11-A, the non-overlap spectrum caused by Doppler centroid frequency difference will not affect the quality of the final interferogram. However, Fig. 8(a) is much noisier than all other interferograms. This is because the non-overlap spectrum caused by burst misalignment is not removed and affects the quality of the final interferogram.

\section{Nepal Earthquake Example}

The Mw 7.8 Gorkha Earthquake in Nepal struck on Apr. 25, 2015 and has attracted wide interest among the geophysical community [19], [20]. We measure the largescale land deformation caused by the Gorkha earthquake using the proposed processing workflow.

We first process a ScanSAR-ScanSAR pair acquired on Feb. 22, 2015 and May 3, 2015 on descending track 48, so the pair were acquired after burst overlap was controlled by JAXA. We processed 2 frames. A comparison of the subswath offsets of the lower frame from coregistration and calculated from parameters is shown in Table IV. There is no big Doppler centroid frequency difference between the pair. Our burst overlap calculation results show that, the burst overlap of subswath 1 to 5 are $66.8 \%$, $68.7 \%, 70.5 \%, 71.9 \%$ and $74.7 \%$, respectively. The increase of burst overlap in terms of percentage is mainly caused by the increase of burst length. If we convert burst misalignment to the number of $1 / \mathrm{PRF}$ of subswath 1 , the results are $118.86,118.14$, $116.81,116.94$ and 116.25 , respectively. The numbers are very close, and the differences are small compared with burst lengths, which relatively tests the estimation precision of the start times of raw bursts.

A comparison of interferograms with and without MBF filtering is shown in Fig. 9. From this figure, we can see that after MBF filtering, the interferogram is greatly improved. The poorer quality of the original interferogram is mainly caused by the non-overlap spectrum caused by burst misalignment. The final unwrapped differential interferogram maps the large-scale land deformation caused by the Gorkha earthquake and is shown in Fig. 10. The long-wavelength signal of the interferogram is caused by ionosphere [25]. 
We then process a ScanSAR-stripmap pair of ascending track 157. In all, two subswaths of one ScanSAR frame and four stripmap frames are processed to cover this large earthquake as much as possible. The stripmap and ScanSAR data were acquired on Jul. 25, 2015 and Aug. 9, 2014, respectively. On this track, the only ScanSAR-ScanSAR pair we have includes scenes acquired before and after burst overlap was controlled, and there is no burst overlap between the pair. Although we have several stripmap acquisitions on this track, the wavelength of the stripmap acquisition we use for ScanSAR-stripmap interferometry is different from that of other stripmap acquisitions.

The MBF filtering result of the stripmap acquisition and its comparison with the ScanSAR acquisition are shown in Fig. 11. A comparison of interferograms with and without MBF filtering is shown in Fig. 12. The final unwrapped interferogram is shown in Fig. 13. Although many frames and subswaths are involved in the processing, the deformation field is not fully covered due to the limited swath width of the stripmap acquisition. Despite this, ScanSAR-stripmap interferograms are useful in that they can increase the number of deformation time series pairs, considering that ALOS-2 routinely acquires data in both ScanSAR and stripmap modes [5].

\section{Conclusion}

In this paper, we present our methods for interferometry with ALOS-2 full-aperture ScanSAR product. We propose a method for estimating the burst length and burst cycle length, and the results for the most widely used ScanSAR mode of ALOS-2 are presented. We propose a method for automatically estimating start times of raw bursts. The successful removal of non-overlap spectra proves the correctness of the estimated parameters.

The MBF we propose can remove non-overlap spectra caused by both Doppler centroid frequency difference and burst misalignment. It is demonstrated to work with both full-aperture ScanSAR images and stripmap SLCs, which enables both ScanSAR-ScanSAR interferometry and ScanSAR-stripmap interferometry. The processing workflow we propose for both ScanSAR-ScanSAR interferometry and ScanSAR-stripmap interferometry based on the MBF can facilitate both programmers and users.

We measure the large-scale land deformation caused by the $2015 \mathrm{Mw} 7.8$ Gorkha earthquake in Nepal using our methods. Both ScanSAR-ScanSAR and ScanSARstripmap interferograms show excellent quality fringes which are very useful for earthquake modeling. On the one hand, this is attributed to the good performance of ALOS- 2 and shows the great potential of ALOS-2 data for large earthquake study. On the other hand, this also demonstrates the performance of our methods.

\section{Acknowledgment}

This research was carried out at the Jet Propulsion Laboratory (JPL), California Institute of Technology, under a contract with the National Aeronautics and Space Administration (NASA Earth Surface and Interior focus area). This research was also supported by an appointment to the NASA Postdoctoral Program at JPL. The ALOS-1 data are provided by Japan Aerospace Exploration Agency (JAXA) through Alaska Satellite Facility (ASF). The ALOS-2 original data are copyright JAXA and provided under JAXA RA4 PI projects P1372002 and P1385002. We thank Piyush Agram (JPL) for his geometrical coregistration programs, and David T. Sandwell and Eric 
Lindsey (University of California, San Diego) for discussions about ALOS-2. We thank the three reviewers for their comments that greatly improve the focus and clarity of this paper.

\section{References}

[1] K. Tomiyasu, "Conceptual performance of a satellite borne, wide swath synthetic aperture radar," IEEE Trans. Geosci. Remote Sens., vol. GE-19, no. 2, pp. 108116, Apr. 1981.

[2] R. K. Moore, J. P. Claassen, and Y. H. Lin, "Scanning spaceborne synthetic aperture radar with integrated radiometer," IEEE Trans. Aerosp. Electron. Syst., vol. AES-17, no. 3, pp. 410-420, May 1981.

[3] Y. Kankaku, Y. Osawa, S. Suzuki, and T. Watanabe, "The overview of the L-band SAR onboard ALOS-2," in Proc. PIERS, Moscow, Russia, Aug. 18-21, 2009, pp. 735-738.

[4] Y. Kankaku, "ALOS-2 and PALSAR-2 development status," presented in Cal-Val Science Team (CVST) Meeting.

[5] M. Shimada, "ALOS/ALOS-2 contribution to GEOSS," presented in The 7th GEOSS Asia-Pacific Symposium, Tokyo, Japan, May 26-28, 2014.

[6] R. Bamler and M. Eineder, "ScanSAR processing using standard high precision SAR algorithms," IEEE Trans. Geosci. Remote Sens., vol. 34, no. 1, pp. 212-218, Jan. 1996.

[7] A. Monti Guarnieri and C. Prati, "ScanSAR focusing and interferometry," IEEE Trans. Geosci. Remote Sens., vol. 34, no. 4, pp. 1029-1038, July 1996.

[8] I. G. Cumming and F. H. Wong, Digital Processing of Synthetic Aperture Radar Data: Algorithms and Implementation. Norwood, MA, USA: Artech House, 2005.

[9] C. Liang, Q. Zeng, J. Jia, J. Jiao, and X. Cui, "ScanSAR interferometric processing using existing standard InSAR software for measuring large scale land deformation," Comput. Geosci., vol. 51, pp. 439-448, Feb. 2013.

[10] E. J. Fielding, A. Sladen, Z. Li, J.-P. Avouac, R. Bürgmann, and I. Ryder, "Kinematic fault slip evolution source models of the 2008 M7.9 Wenchuan earthquake in China from SAR interferometry, GPS and teleseismic analysis and implications for Longmen Shan tectonics," Geophys. J. Int., vol. 194, no. 2, pp. 1138-1166, May 2013.

[11] D. Sandwell, R. Mellors, X. Tong, M. Wei, and P. Wessel, "Open radar interferometry software for mapping surface deformation," Eos Trans. AGU, vol. 92, no. 28, pp. 234-235, Jul. 2011.

[12] E. Sansosti, P. Berardino, M. Manunta, F. Serafino, and G. Fornaro, "Geometrical SAR image registration," IEEE Trans. Geosci. Remote Sens., vol. 44, no. 10 , pp. 2861-2870, Oct. 2006.

[13] R. Scheiber and A. Moreira, "Coregistration of interferometric SAR images using spectral diversity," IEEE Trans. Geosci. Remote Sens., vol. 38, no. 5, pp. 2179-2191, Sep. 2000.

[14] P. A. Rosen, S. Hensley, G. Peltzer, and M. Simons, "Updated repeat orbit interferometry package released," EOS Trans. $A G U$, vol. 85, no. 5, p. 47, 2004.

[15] P. A. Rosen, E. Gurrola, G. F. Sacco, and H. Zebker, "The InSAR scientific computing environment," in Proc. EUSAR, Nuremberg, Germany, Apr. 23-26, 2012, pp. 730-733.

[16] C. Liang and E. Fielding, "Interferometric processing of ScanSAR data using stripmap processor: New insights from coregistration," IEEE Trans. Geosci. Remote Sens., vol. 54, no. 7, pp. 4343-4354, Jul. 2016. 
[17] E. O. Lindsey, R. Natsuaki, X. Xu, M. Shimada, M. Hashimoto, D. Melgar, and D. T. Sandwell, "Line of sight displacement from ALOS-2 interferometry: Mw 7.8 Gorkha earthquake and Mw 7.3 aftershock," Geophys. Res. Lett., vol. 42, no. 16, pp. 6655-6661, Aug. 2015.

[18] C. Liang, "Interferogram for ALOS2-track 48-swath ScanSARNominal14MHz; Feb 22, 2015 to May 3, 2015," UNAVCO InSAR Product, doi: 10.7283/S2KW2R

[19] J. Galetzka et al., "Slip pulse and resonance of the Kathmandu basin during the 2015 Gorkha earthquake, Nepal," Science, vol. 349, no. 6252, pp. 1091-1095, Sep. 2015.

[20] J. S. Kargel et al., "Geomorphic and geologic controls of geohazards induced by Nepal's 2015 Gorkha earthquake," Science, vol. 351, no. 6269, pp. 140-150, Jan. 2016.

[21] J. Holzer and R. Bamler, "Burst-mode and ScanSAR interferometry," IEEE Trans. Geosci. Remote Sens., vol. 40, no. 9, pp. 1917-1934, Sep. 2002.

[22] C. Liang, Q. Zeng, J. Jiao, and X. Cui, "On the phase compensation of short ScanSAR burst focused by long matched filter for interferometric processing," IEEE Trans. Geosci. Remote Sens., vol. 52, no. 2, pp. 1299-1310, Feb. 2014.

[23] JAXA, ALOS-2/PALSAR-2 Level 1.1/1.5/2.1/3.1 CEOS SAR Product Format Description.

[24] C. Liang, Q. Zeng, and J. Jiao, "An assessment of ScanSAR interferometric processing using full-aperture approach," IEEE Geosci. Remote Sens. Lett., vol. 11, no. 9, pp. 1559-1563, Sep. 2014.

[25] C. Liang and E. J. Fielding, "Measuring azimuth deformation with L-band ALOS-2 ScanSAR interferometry," IEEE Trans. Geosci. Remote Sens., in press. 


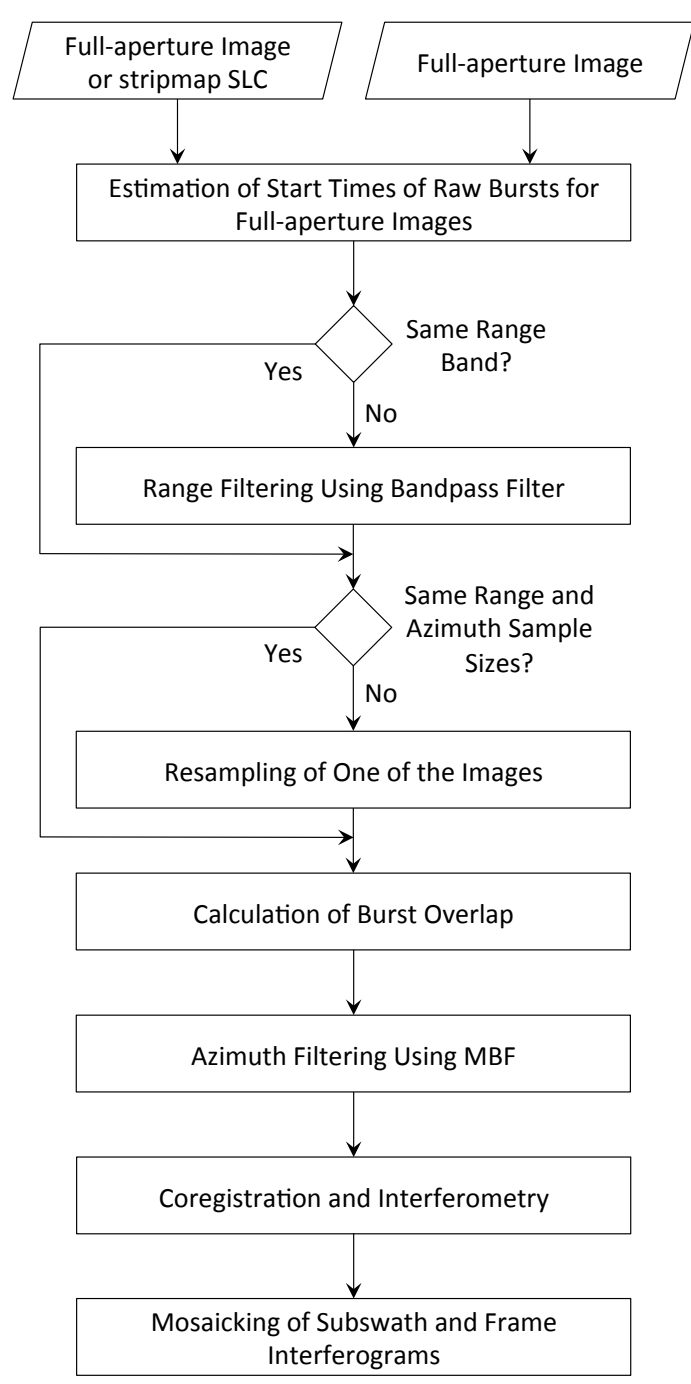

Fig. 1. A processing workflow for both ScanSAR-ScanSAR interferometry and ScanSAR-stripmap interferometry with ALOS-2 full-aperture ScanSAR product.

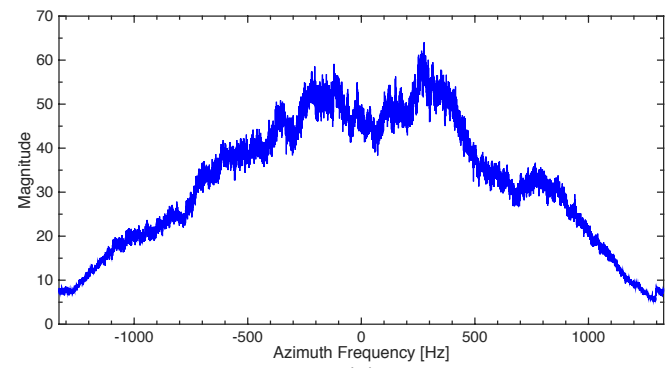

(a)

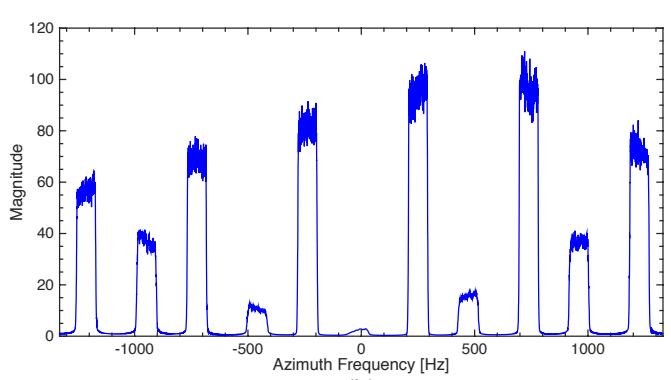

(b)

Fig. 2. Azimuth spectrum of full-aperture ScanSAR image. (a) Spectrum of original image. (b) Spectrum of deramped image. The azimuth duration of the data used is about full-aperture length. In (b), signals of some bursts are outside the PRF interval and shifted to the PRF interval due to aliasing effect. Data: subswath 1 of ALOS-2 full-aperture product acquired on Aug. 9, 2014. 


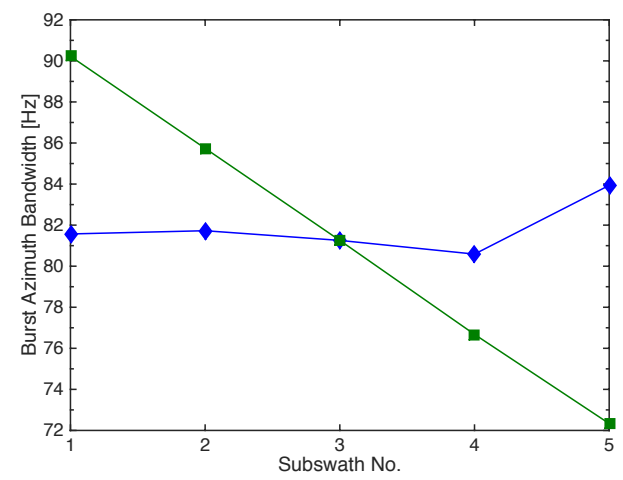

Fig. 3. Burst azimuth bandwidth of ALOS-2 WBD mode. Blue line with diamonds represents swath center bandwidth of each subswath calculated using each subswath's burst length. Green line with squares represents swath center bandwidth of each subswath calculated using subswath 3's burst length. Data: frame 0550 acquired on Aug. 9, 2014.

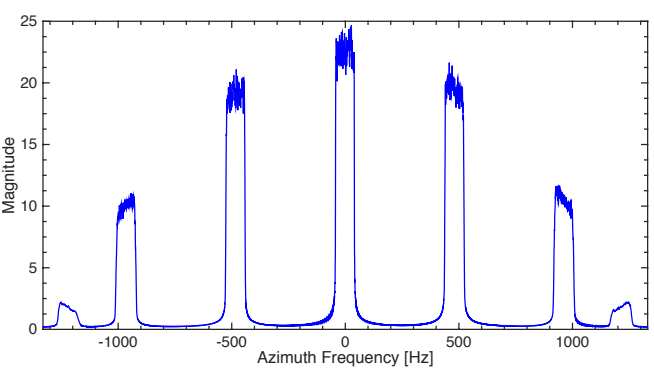

(a)

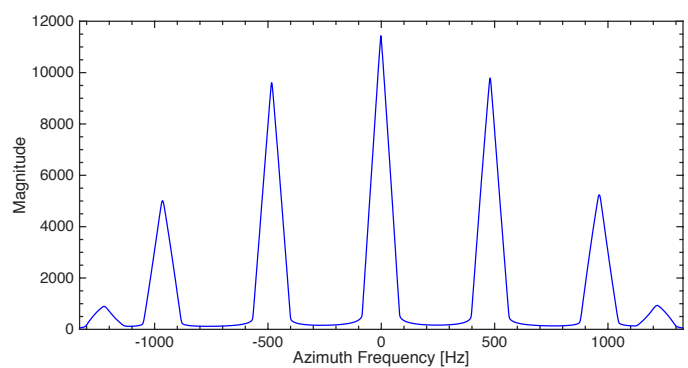

(b)

Fig. 4. (a) Azimuth spectrum of full-aperture ScanSAR image. (b) Correlation of the magnitude of the spectrum and the magnitude of an artificial burst spectrum with bandwidth equal to $K_{a} T_{B}$. The azimuth duration of the data used is burst cycle length $T_{C}$. Note that there is only very slight aliasing effect compared with Fig. 1(b). Data: subswath 1 of ALOS-2 full-aperture product acquired on Aug. 9, 2014. 


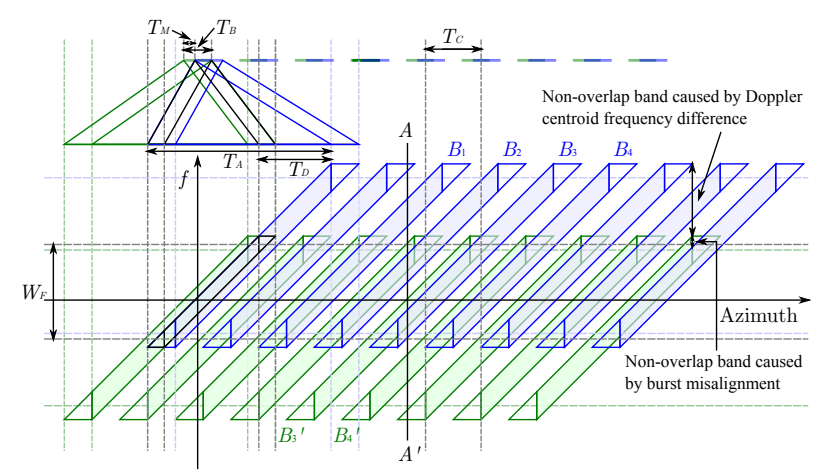

(a)

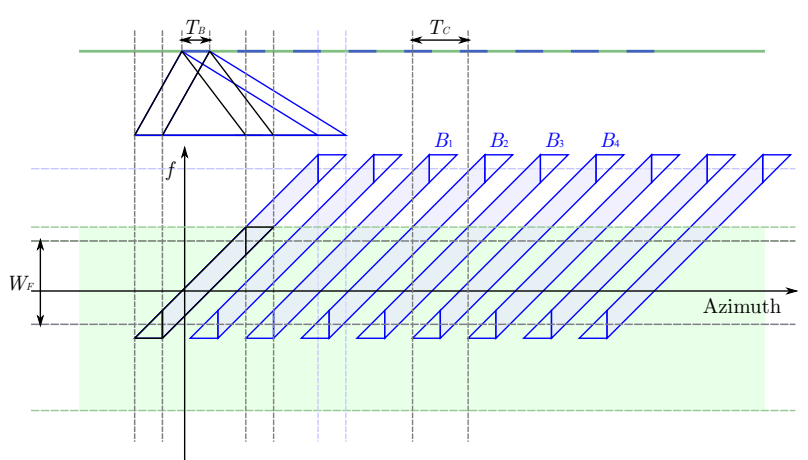

(b)

Fig. 5. Spectrum of interferometry with full-aperture image. (a) ScanSAR-ScanSAR interferometry. (b) ScanSAR-stripmap interferometry. Green and light green denote one acquisition. Blue and light blue denote the other acquisition. Black and gray denote the effective ScanSAR mode for the InSAR pair which corresponds to the overlap spectrum. $T_{A}$ is the full aperture length. $T_{D}$ is the time difference corresponding to the Doppler centroid frequency difference. $T_{B}$ is the burst length. $T_{M}$ is the burst misalignment. $T_{C}$ represents the burst cycle to be filtered for the acquisition denoted by blue and light blue, and bursts $B_{1}-B_{4}$ are involved in the filtering. Bursts $B_{3}^{\prime}$ and $B_{4}^{\prime}$ correspond to bursts $B_{3}$ and $B_{4}$, respectively. $W_{F}$ (Cutoff frequency corresponds to burst center) is the full azimuth bandwidth of the effective ScanSAR mode. Line $A A^{\prime}$ indicates the azimuth spectra of a target. To make the figure less complicated, only the acquisition geometries of the first bursts are plotted, and only the first effective burst is highlighted by black.
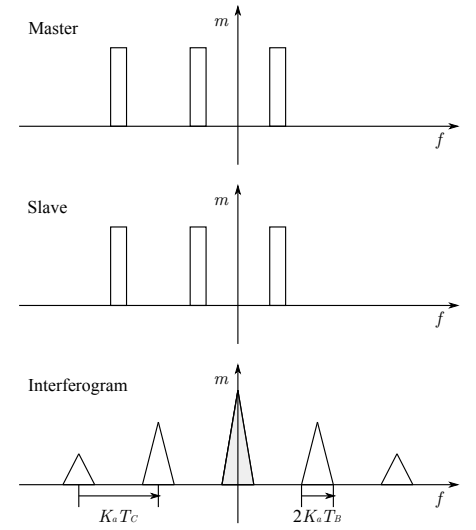

(a)
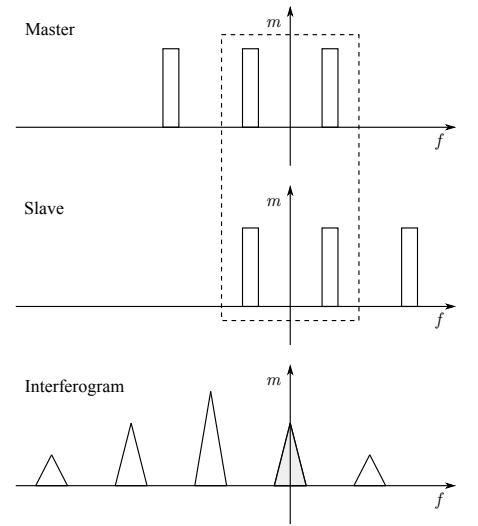

(b)
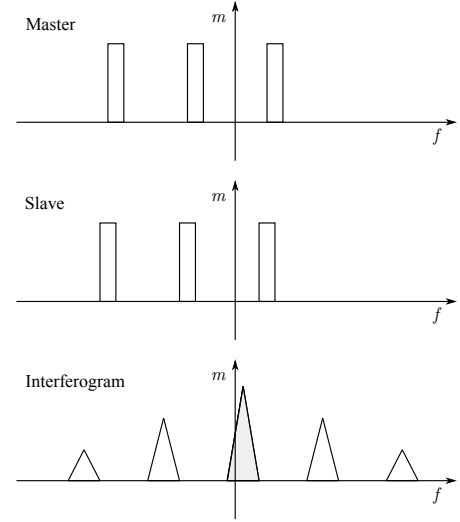

(c) 
Fig. 6. Spectra correlation of a target during interferometry with full-aperture image. (a) No Doppler centroid frequency difference or burst misalignment. (b) With Doppler centroid frequency difference. (c) With burst misalignment. In (b), only bursts inside the rectangle are corresponding (coherent) bursts.
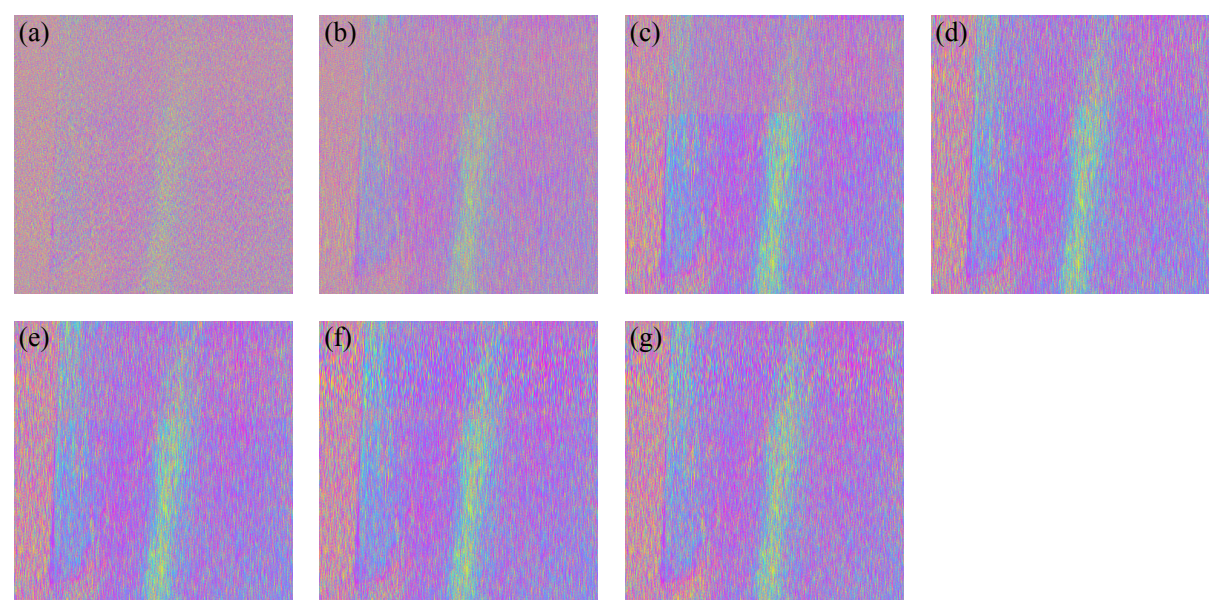

Fig. 7. Full-aperture interferograms without sample averaging and downsampling. (a) is the original interferogram. (b)-(f) are interferograms after removing non-overlap spectrum caused by burst misalignment using MBF. Non-overlap spectrum caused by Doppler centroid frequency difference is also removed for (c)-(f) using different starting sample in the MBF filtering. For the starting sample, the starting sample of (c) is opposite to the suggested starting sample, (d) always starts from the ending sample of the effective burst, (e) always starts from the starting sample of the effective burst, and (f) uses the suggested starting sample. (g) is the interferogram generated by processing data of the effective ScanSAR mode starting from raw data.
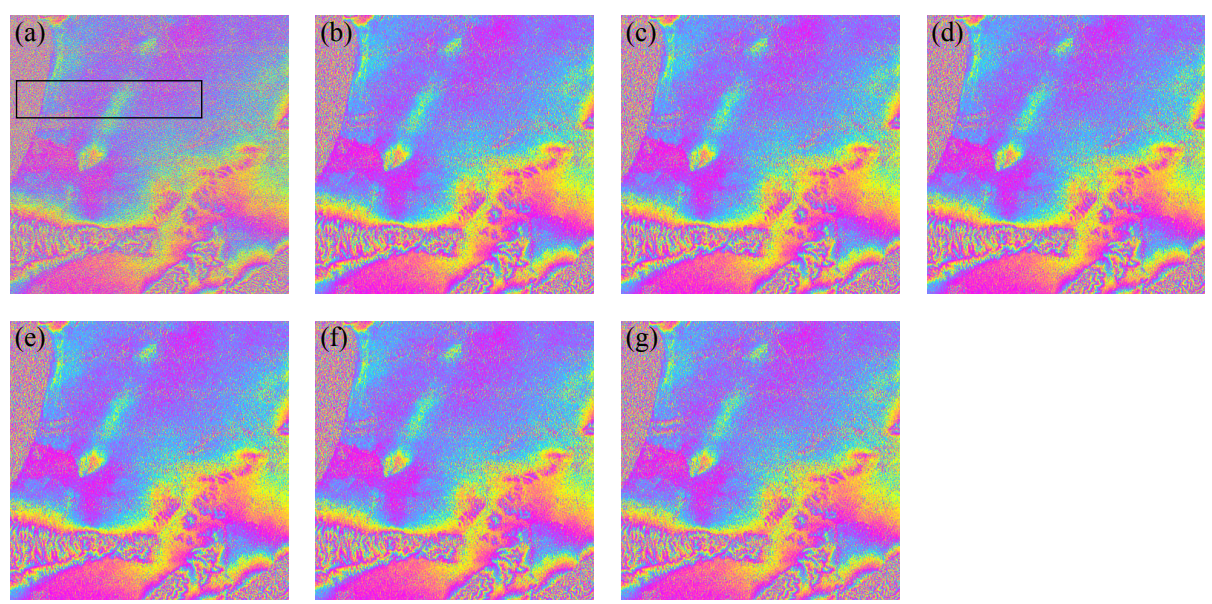

Fig. 8. Full-aperture interferograms with sample averaging and downsampling. (a)-(g) correspond to Fig. 7(a)-(g). The black rectangle indicates the area shown in Fig. 7. 


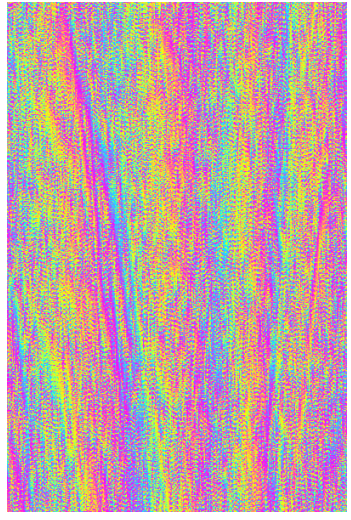

(a)



(b)

Fig. 9. ScanSAR-ScanSAR interferogram before sample averaging. (a) Interferogram produced using images without MBF filtering. (b) Interferogram produced using images with MBF filtering. The "spikes" of the interferograms will be removed in the subsequent azimuth sample averaging. 

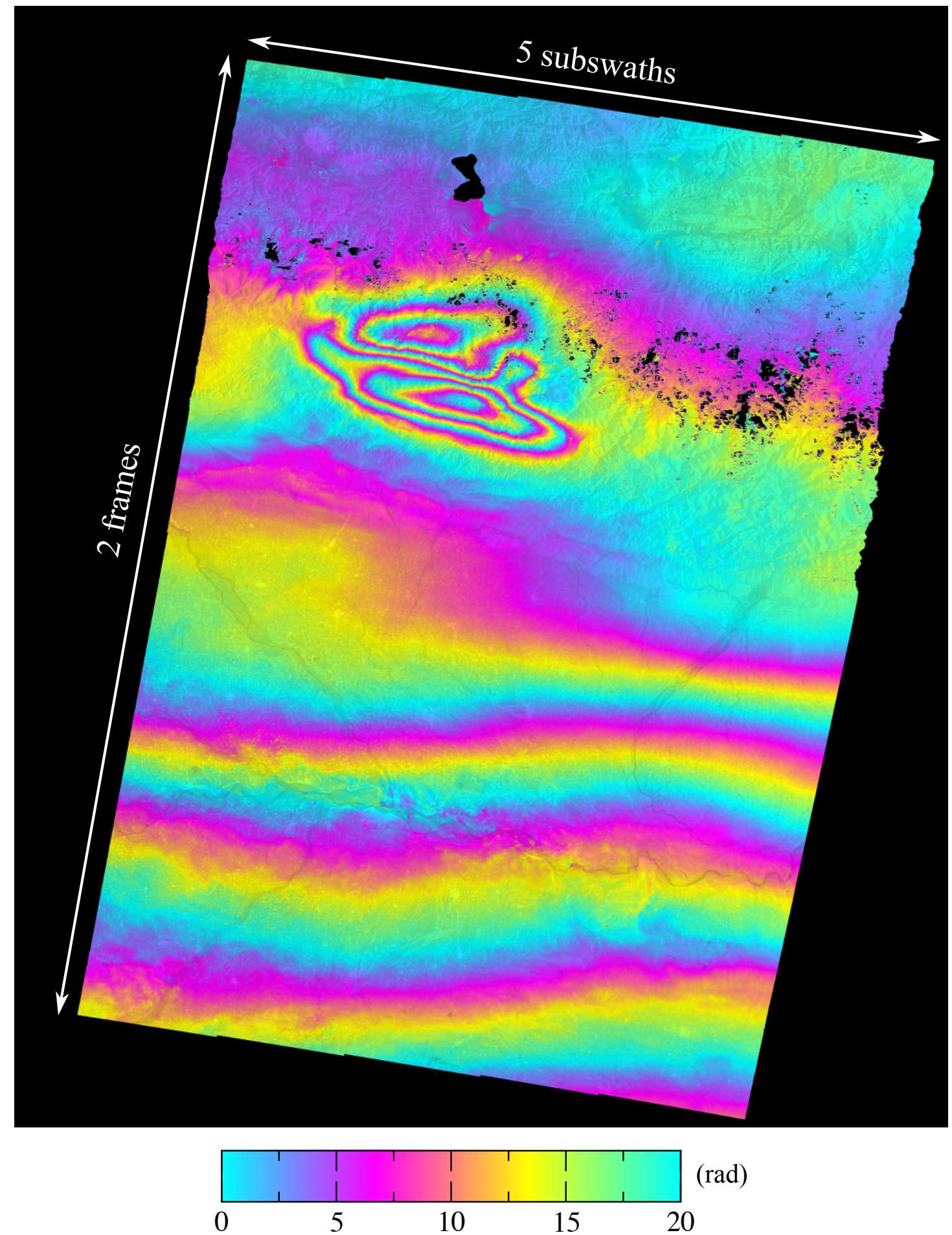

$(\mathrm{rad})$

Fig. 10. ScanSAR-ScanSAR interferogram showing the large-scale land deformation caused by Nepal earthquake. The interferogram is rewrapped after phase unwrapping, and superimposed on the amplitude image. 


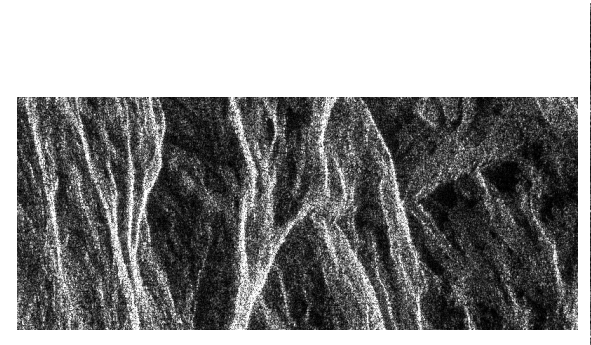

(a)

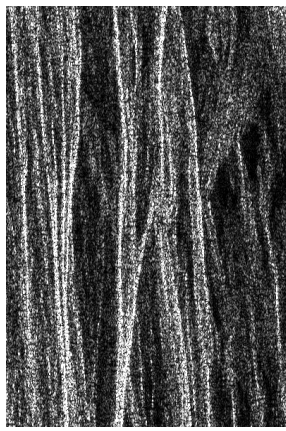

(b)

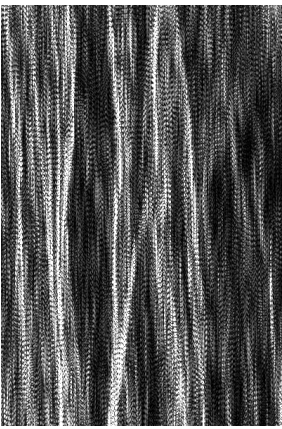

(c)



(d)

Fig. 11. (a) Original stripmap SLC. (b) Stripmap SLC after range filtering and resampling. (c) Stripmap SLC after range filtering, resampling and MBF filtering. (d) Full-aperture ScanSAR image of the same area.

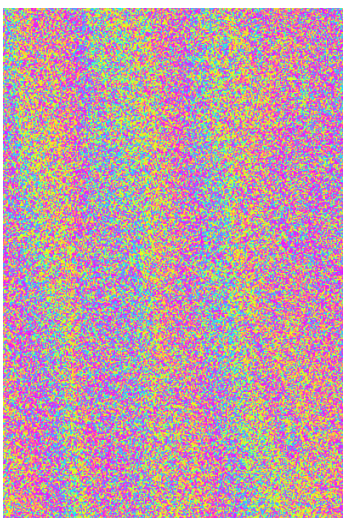

(a)

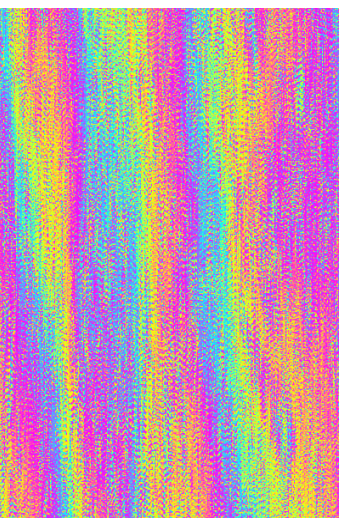

(b)

Fig. 12. ScanSAR-stripmap interferogram before sample averaging. (a) Interferogram produced using images without MBF filtering. (b) Interferogram produced using images with MBF filtering. The "spikes" of the interferogram in (b) will be removed in the subsequent azimuth sample averaging. 


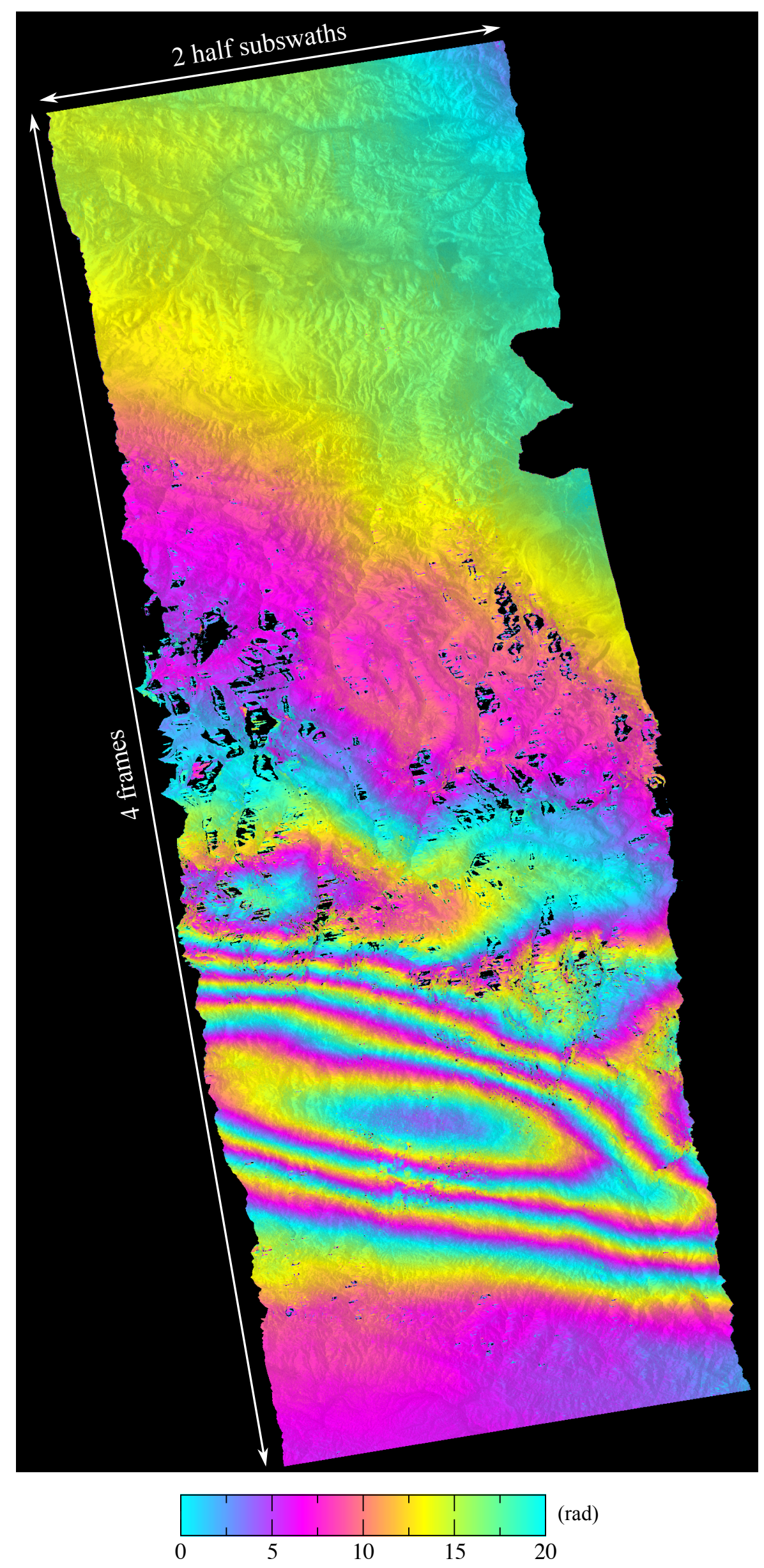


Fig. 13. ScanSAR-stripmap interferogram showing the large-scale land deformation caused by Nepal earthquake. The interferogram is rewrapped after phase unwrapping, and superimposed on the amplitude image.

Table I Burst Length and Burst Cycle Length of ALOS-2 WBD Mode (Number of Each Subswath's 1/PRF)

\begin{tabular}{lccccc}
\hline Parameter & S1 & S2 & S3 & S4 & S5 \\
\hline PRF & 2661.847 & 3314.512 & 2406.568 & 2270.575 & 2821.225 \\
Burst Length & 358 & 470 & 358 & 355 & $487 *$ \\
Burst Cycle Length & 2086.26 & 2597.80 & 1886.18 & 1779.60 & 2211.17 \\
\hline
\end{tabular}

*burst length may subject to small change for subswath 5. This also affects burst cycle length.

Table II Burst Length and Burst Cycle Length of ALOS-2 WBD Mode (Number of Subswath 1's 1/PRF)

\begin{tabular}{lccccc}
\hline Parameter & S1 & S2 & S3 & S4 & S5 \\
\hline PRF & 2661.847 & 3314.512 & 2406.568 & 2270.575 & 2821.225 \\
Burst Length & 358.00 & 377.45 & 395.98 & 416.17 & $459.49^{*}$ \\
Burst Cycle Length & \multicolumn{5}{c}{2086.26} \\
burst length may subject to small change for subswath 5. This also affects burst cycle length.
\end{tabular}

Table III Parameters Used for Simulation

\begin{tabular}{lll}
\hline Parameter & Value & \\
\hline Data Acquisition Dates & $2007-07-03$ & $2007-10-03$ \\
Burst Length [Number of Echoes] & 307 & \\
Burst Gap Length [Number of Echoes] & 1537 & \\
Number of Misaligned Echoes & 153.18 \\
PRF [Hz] & 2159.83 & \\
Azimuth Bandwidth [Hz] & 1403.89 & -242.12 \\
Doppler Centroid Frequency [Hz] & 401.94 & \\
Approximate Number of Looks of the Original ScanSAR Mode & 3.10 & \\
Approximate Number of Looks of the Effective ScanSAR Mode & 1.69 & \\
\hline
\end{tabular}

Table IV Offsets Between Subswaths. Offsets Are Measured in Subswath 1's Sample Sizes. Data: Frame 3100 Acquired on Feb. 22, 2015.

\begin{tabular}{lcccc}
\hline Paramter & S1-S2 & S2-S3 & S3-S4 & S4-S5 \\
\hline Range Offset from Parameters & -4367.941 & -4090.983 & -5300.999 & -5391.998 \\
Range Offset from Coregistration & -4368.674 & -4089.677 & -5302.351 & -5392.007 \\
Range Offset Difference & 0.733 & -1.306 & 1.352 & 0.009 \\
\hline Azimuth Offset from Parameters & -351.569 & -378.437 & -396.115 & -418.123 \\
Azimuth Offset from Coregistration & -350.823 & -378.901 & -395.482 & -417.353 \\
Azimuth Offset Difference & -0.746 & 0.463 & -0.632 & -0.770 \\
\hline
\end{tabular}

